\title{
Identification of an Cysteine-to-Arginine Substitution Caused by a Single Nucleotide Polymorphism in the Canine Monoamine Oxidase B Gene
}

\author{
Chie HASHIZUME ${ }^{1}$, Koji MASUDA $^{1)}$, Yukihide MOMOZAWA ${ }^{1)}$, Takefumi KIKUSUI ${ }^{1}$, Yukari TAKEUCHI $^{1) *}$ and \\ Yuji MORI ${ }^{1)}$ \\ ${ }^{1)}$ Laboratory of Veterinary Ethology, The University of Tokyo, Tokyo 113-8657, Japan
}

(Received 20 August 2004/Accepted 15 October 2004)

\begin{abstract}
Monoamine oxidase B catalytically oxidizes biogenic amines such as phenylethylamine and dopamine, and its activity is presumed to be related to particular behavioral traits. In this study, we first identified a single nucleotide polymorphism (T199C) located on the putative third exon of the canine monoamine oxidase B gene, which causes an amino acid substitution from cysteine to arginine. We then examined the allelic frequencies in five dog breeds (Golden Retriever, Labrador Retriever, Maltese, Miniature Schnauzer, and Shiba) and found significant variation among them. The present results suggest that analysis of the monoamine oxidase B polymorphism could be a useful means of elucidating the genetic background of breed-specific behavioral characteristics in dogs. KEY WORDS: canine, MAOB, polymorphism.
\end{abstract}

J. Vet. Med. Sci. 67(2): 199-201, 2005

Canine monoamine oxidase $\mathrm{B}$ (MAOB) is a pivotal enzyme that catalytically oxidizes monoamines in both the brain and peripheral tissues. In the canine brain, MAOB is located mainly in the hypothalamus and hippocampus [6]. In human studies, relationships between low levels of platelet MAOB activity and particular personality traits such as sensation-seeking, monotony avoidance, impulsiveness, and aggression have been reported $[3,7,10]$.

A single nucleotide polymorphism (SNP) located on intron 13 of the human MAOB gene was reported to be associated with MAOB activity in both the brain [1] and platelets [2]. In this study, we investigated the entire region of MAOB complementary DNA (cDNA) in an attempt to identify SNPs in the canine MAOB gene. Then, the identified SNP was examined for its genetic diversity among five canine breeds (Golden Retriever, Labrador Retriever, Maltese, Miniature Schnauzer, and Shiba) by genotyping its allelic frequencies.

In order to search for any polymorphic site that might be present in the MAOB gene (GenBank accession: $\mathrm{AB}$ 070958), we first amplified the nucleotide sequence for the whole amino acid coding region of the MAOB gene, using the brain cDNA of eleven Beagles as the template; to this end, RT-PCR was carried out according to a method we described in a previous report [6]. DNA sequencing was performed with the BigDye Terminator Cycle Sequencing FS Ready Reaction Kit using the ABI PRISM 377 DNA sequencer (Perkin-Elmer, U.S.A.). In order to assess genetic variation in the putative polymorphic region, blood cell samples were obtained from 210 individuals of the 5 dog breeds (46 Labrador Retrievers, 57 Golden Retrievers, 35 Malteses, 27 Miniature Schnauzers, and 45 Shibas). These blood samples were taken with the owners' consent

\footnotetext{
* CoRRespondence to: TAKeUChi, Y., Laboratory of Veterinary Ethology, The University of Tokyo, 1-1-1 Yayoi, Bunkyo-ku, Tokyo 113-8657, Japan.
}

during regular health checks in veterinary clinics. Genomic DNA was extracted with the QIAamp Blood Midi Kit (QIAGEN, U.S.A.), dissolved into $\mathrm{H}_{2} \mathrm{O}$, and stored at $4^{\circ} \mathrm{C}$ until PCR was performed. To amplify the region containing the identified SNP (T199C), oligonucleotide primers for PCR were designed. The sequence of the forward primer was 5'-TCCATGGATACACCTCAAGG-3', and the reverse primer was 5'-TGATGGATGAGACGCTCTAC3 '. PCR was performed with $30 \mathrm{ng}$ of genomic DNA in a $100 \mu l$ reaction volume, which consisted of $200 \mu \mathrm{M}$ of dNTPs, $10 \mu l 10 \times$ Ex Taq Buffer (Takara, Japan), $0.3 \mu \mathrm{M}$ of each primer, and 1.25 units of TaKaRa Ex Taq (Takara, Japan). After an initial denaturation at $95^{\circ} \mathrm{C}$ for $5 \mathrm{~min}, \mathrm{PCR}$ was performed using 35 successive cycles of $95^{\circ} \mathrm{C}$ for 30 sec, $60^{\circ} \mathrm{C}$ for $30 \mathrm{sec}$, and $72^{\circ} \mathrm{C}$ for $30 \mathrm{sec}$. Chain elongation at $72^{\circ} \mathrm{C}$ was extended to $5 \mathrm{~min}$ after the final cycle. The PCR product $(44 \mu l)$ was then digested with 8 units of Hinf I enzyme (Takara, Japan) in a total volume of $50 \mu l$ under conditions recommended by the manufacturer. The products were then electrophoresed on $3.0 \%$ agarose gel in order to detect the allelic pattern, and the inter-breed allelic frequencies and the fitness to the Hardy-Weinberg equilibrium were calculated using the $\chi^{2}$ test.

A SNP was identified to be located putatively on the third exon of the canine MAOB gene by comparing the obtained cDNA sequences, which suggested an amino acid substitution from cysteine to arginine at the 67th residue from the first methionine. The identified DNA sequences of exon 3 $(137 \mathrm{bp})$ were identical to those obtained from the canine whole genome shotgun sequence that has recently (July 2004) been made available (GenBank; AAEX01047807: Canis familiaris chromosome X, cont_47806).

All of the individuals investigated in this study $(n=210)$ were successfully genotyped by the PCR-RFLP method. If nucleotide $\mathrm{C}$ occupied the polymorphic site, digestion of the PCR product by Hinf I generated two fragments, i.e., 180 


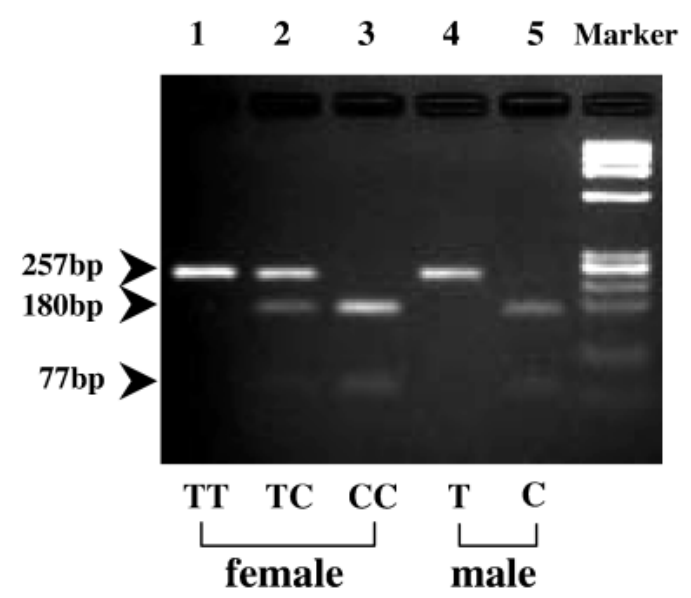

Fig. 1. Canine MAOB T199C polymorphism demonstrated by PCR-RFLP and electrophoresis. The bands are visualized by ethidium bromide staining. The genotypes are 1: TT, 2: TC, 3: CC (female), 4: T and 5: C (male).

and $77 \mathrm{bp}$ (allele C). However, the PCR product containing nucleotide $\mathrm{T}$ at the polymorphic site (allele $\mathrm{T}$ ) was not cleaved by Hinf I (Fig. 1). The allele frequencies were found to fit the Hardy-Weinberg equilibrium. Statistical analyses revealed a significant difference in allele distribution among breeds (male: $\chi^{2}=38.9, \mathrm{df}=4, \mathrm{p}<0.0001$; female: $\left.\chi^{2}=39.9, \mathrm{df}=4, \mathrm{p}<0.0001\right)$. The observed allelic frequency of the $\mathrm{T}$ allele differed between Golden Retrievers (over 90\%) and Miniature Schnauzers (less than 17\%) in both sexes. Shibas exhibited the highest value (50.8\%) for heterozygosity in the MAOB gene, while Golden Retrievers showed the lowest value $(17.7 \%$ ) among the 5 breeds (Table $1)$.

The enzyme MAOB is thought to play a major role in the oxidation of monoamines in cooperation with the co-factor FAD [12], and the crucial region for FAD binding has been assumed to be located between the 62nd and 103rd amino acids from the first methionine in humans [4]. We previously reported that the FAD binding site of the canine MAOB gene was identical to the human MAOB gene [6]; based on the results of that study and other observations, we now estimate that the crucial region for FAD binding in the canine MAOB gene is also identical to that of the human MAOB gene. The amino acid substitution caused by this SNP is thought to be located on the 67 th residue, which also appears to be an important site for FAD binding in the canine MAOB gene. Because the amino acid residues of 67 th for human and rat MAOB were reported to be arginine, it could be important for FAD binding rather than cysteine. If this is indeed the case, the identified SNP, which causes an amino acid substitution, may lead to a structural change in the MAOB protein and thereby affect the efficacy of the oxidative deamination of monoamines.

In this study, significant differences in the allelic frequency among breeds in both males and females were
Table 1. Allele frequency and heterozygosity of MAOB SNP (T199C) in five dog breeds

\begin{tabular}{lcrrr}
\hline Male & $\mathrm{n}$ & $\mathrm{C}$ & $\mathrm{T}$ & \\
\hline Golden Retriever & 31 & $2(6.5)$ & $29(93.5)$ & \\
Labrador Retriever & 20 & $6(30.0)$ & $14(70.0)$ & \\
Maltese & 26 & $14(53.8)$ & $12(46.2)$ & \\
Miniature Schnauzer & 15 & $14(93.3)$ & $1(6.7)$ & \\
Shiba & 29 & $18(62.1)$ & $11(37.9)$ & \\
& & & & \\
Female & $\mathrm{n}$ & $\mathrm{C}$ & $\mathrm{T}$ & Heterozygosity \\
\hline Golden Retriever & 26 & $5(9.6)$ & $47(90.4)$ & 0.177 \\
Labrador Retriever & 26 & $23(44.2)$ & $29(55.8)$ & 0.503 \\
Maltese & 19 & $14(36.8)$ & $24(63.2)$ & 0.478 \\
Miniature Schnauzer & 12 & $20(83.3)$ & $4(16.7)$ & 0.290 \\
Shiba & 16 & $14(43.8)$ & $18(56.2)$ & 0.508 \\
\hline
\end{tabular}

The percentage is shown in parentheses.

observed. In particular, more than $90 \%$ of the Golden Retrievers had allele T, whereas less than $17 \%$ of the Miniature Schnauzers had allele T. This discrepancy in allelic frequency among the breeds is perhaps the result of selective breeding, as was demonstrated previously in the case of the canine DRD4 gene [9].

Golden Retrievers and Miniature Schnauzers have notably different characteristics such as body size, coat color, and a number of behavioral traits. In a classic study of breed differences with respect to behavioral traits, Golden Retrievers were assessed as being a calm and friendly breed, whereas Miniature Schnauzers are an easily excited and/or aggressive breed [5]. In our previous study, the canine MAOB gene was found to be expressed mainly in the hypothalamus and hippocampus [6]. These brain regions are thought to be involved in the control of emotional states, including aggression [8]. Moreover, low levels of homovanillic acid (HVA), a metabolite of dopamine, in the cerebrospinal fluid was reported in dogs that had exhibited severe impulsive aggression [11]. Based upon these findings, it appears reasonable to speculate that some breed-specific behavioral traits can be ascribed, at least in part, to certain SNPs, including the one in the MAOB gene described in the present study. Further investigation is needed to verify the hypothesis of the relationship between MAOB and breed-specific behavioral traits, as such information will facilitate our understanding of various aspects of behavior traits in canine species.

ACKNOWLEDGMENTS. We are indebted to DVMs Norio Kogure, Kazue Igarashi, Hisao Imoto, Reiko Usui, Keiko Uchida, Tetsuyasu Uno, Ayako Kakinuma, Shoji Satoh, Masami Takebe, Makoto Tatemetsu, and Kaori Murata and the staff of their veterinary hospitals for their kind cooperation in collecting blood samples from dogs. This work was supported by grants-in-aid for scientific research from the Ministry of Education, Science, Sports, Culture and Technology (03460131). 


\section{REFERENCES}

1 Balciuniene, J., Emilsson, L., Oreland, L., Pettersson, U. and Jazin, E. 2002. Hum. Genet. 110: 1-7.

2 Garpenstrand, H., Ekblom, J., Forslund, K., Rylander, G. and Oreland, L. 2000. J. Neural. Transm. 107: 523-530.

3 Garpenstrand, H., Longato-Stadler, E., af Klinteberg, B., Grigorenko, E., Damberg, M., Oreland, L. and Hallman, J. 2002. Eur. Neuropsychopharmacol. 12: 135-140.

4 Gottowik, J., Malherbe, P., Lang, G., Da Prada, M. and Cesura, A. M. 1995. Eur. J. Biochem. 230: 934-942.

5 Hart, B. L. and Miller, M. F. 1985. J. Am. Vet. Med. Assoc. 186: $1175-1180$.

6 Hashizume, C., Masuda, K., Momozawa, Y., Kikusui, T.,
Takeuchi, Y. and Mori, Y. 2003. J. Vet. Med. Sci. 65: 893-898.

7 Klinteberg, B., Schalling, D., Edman, G., Oreland, L. and Asberg, M. 1987. Neuropsychobiology 18: 89-96.

8 Krotewicz, M. and Romaniuk, A. 1995. Acta. Neurobiol. Exp. (Warsz) 55: 271-279.

9 Niimi, Y., Inoue-Murayama, M., Kato, K., Matsuura, N., Murayama, Y., Ito, S., Momoi, Y., Konno, K. and Iwasaki, T. 2001. J. Hered. 92: 433-436.

10 Oreland, L. and Hallman, J. 1995. Prog. Brain Res. 106: $77-$ 84.

11 Reisner, I. R., Mann, J. J., Stanley, M., Huang, Y. Y. and Houpt, K. A. 1996. Brain Res. 714: 57-64.

12 Shih, J. C., Chen, K. and Ridd, M. J. 1999. Annu. Rev. Neurosci. 22: 197-217. 